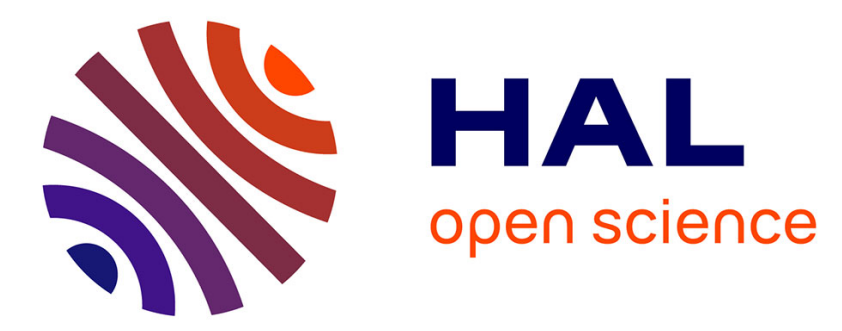

\title{
Markovian Bridge Maintenance Planning Incorporating Corrosion Initiation and Nonlinear Deterioration
}

\author{
Alan J O'Connor, Emma Sheils, Denys Breysse, Franck Schoefs
}

\section{To cite this version:}

Alan J O'Connor, Emma Sheils, Denys Breysse, Franck Schoefs. Markovian Bridge Maintenance Planning Incorporating Corrosion Initiation and Nonlinear Deterioration. Journal of Bridge Engineering, 2013, 18 (3), pp.189-199. 10.1061/(ASCE)BE.1943-5592.0000342 . hal-03283297

\section{HAL Id: hal-03283297 \\ https://hal.science/hal-03283297}

Submitted on 14 Oct 2021

HAL is a multi-disciplinary open access archive for the deposit and dissemination of scientific research documents, whether they are published or not. The documents may come from teaching and research institutions in France or abroad, or from public or private research centers.
L'archive ouverte pluridisciplinaire HAL, est destinée au dépôt et à la diffusion de documents scientifiques de niveau recherche, publiés ou non, émanant des établissements d'enseignement et de recherche français ou étrangers, des laboratoires publics ou privés.

\section{(c)(1)}

Distributed under a Creative Commons Attribution| 4.0 International License 


\title{
Markovian Bridge Maintenance Planning Incorporating Corrosion Initiation and Nonlinear Deterioration
}

\author{
A. J. O’Connor, Ph.D., Aff.M.ASCE ${ }^{1}$; E. Sheils ${ }^{2}$; Denys Breysse ${ }^{3}$; and Franck Schoefs ${ }^{4}$
}

For some materials, such as $\mathrm{RC}$, there is an initiation phase of deterioration where aggressive agents such as chlorides diffuse into the concrete cover. It is necessary to model this initiation time when attempting to determine the optimal maintenance strategy for the lifetime cost associated with a structure. It has been recommended in the literature that Markov chain models should be further improved within maintenance management systems to take initiation time into consideration. Many existing bridge management systems (BMSs), such as BRIDGIT and Pontis, use Markovian-based maintenance management to simulate the propagation phase of deterioration and repair of structures over time. It is therefore useful to continue this progress through incorporation of an initiation phase in relation to the development of Markovian-based maintenance management, which can lead to an improvement in BMSs that are already in place. The incorporation of the initiation phase into deterioration modeling can affect the frequency of inspections, repairs, and failures and hence the expected cost over the remaining lifetime of the structure. On this basis, this issue was addressed as part of this study. A maintenance management model has been further developed to take this two-step deterioration process (i.e., initiation and propagation) into account. This allows owners/managers of bridges to compare the ef-ficiency of different maintenance strategies in terms of both the initiation phase and the propagation phase of deterioration to determine the optimal maintenance strategy for the structure or group of structures being considered. The capabilities of the developed methodology are dem-onstrated using a practical example.

Keywords: Markov; Initiation phase; Deterioration; Maintenance management; Repair.

\section{Introduction}

As bridges age and volumes of traffic continue to increase, bridge maintenance has become a significant challenge for governments/ state agencies around the world that are charged with managing this aging resource. It is now a necessity to derive/develop maintenance management strategies that optimize the use of restricted maintenance budgets and prioritize maintenance actions that provide maximum benefit with respect to both the lifetime cost and the day-to-day performance of the network (Liu and Frangopol 2005; Neves et al. 2004).

To efficiently plan the regime of inspection, maintenance, and repair, it is essential that some form of stochastic deterioration modeling is used to predict the future deterioration state. This modeling must, as a necessity, consider all phases of deterioration of the structures' constituent materials; i.e., in the case of RC and prestressed concrete structures, both the initiation and propagation phases must be modeled. Where available, the results

\footnotetext{
${ }^{1}$ Associate Professor, Dept. of Civil Engineering, Trinity College Dublin, Dublin 2, Ireland (corresponding author). E-mail: alan.oconnor@tcd.ie ${ }^{2}$ Postdoctoral Research Fellow, Urban Institute Ireland, Univ. College Dublin, Dublin 4, Ireland.

${ }^{3}$ Professor, Géosciences, Hydrosciences, Matériaux, Constructions, Univ. Bordeaux 1, 33400 Talence, France.

${ }^{4}$ Professor, Research Institute in Civil and Mechanical Engineering (GeM), UMR 6183, Faculté des Sciences et des Techniques, Univ. of Nantes, F-44000 Nantes, France.
}

of monitoring and inspections can be used to update deterioration models (thereby reducing systemic uncertainty; Corotis et al. 2005).

Bridge management systems (BMSs) have been developed that use Markov chains to simulate the deterioration and repair of structures over time (Corotis et al. 2005; Cesare et al. 1992; Scherer and Glagola 1994; Jiang et al. 2000; Roelfstra et al. 2004; Orcesi and Cremona 2006). In addition, many existing BMSs, such as BRIDGIT and Pontis, use Markov decision process (MDP) models (Rens and Nogueria 2005; Adey et al. 2003; Frangopol et al. 2001). According to Scherer and Glagola (1994), "MDPs are a powerful and useful technique for bridge management systems." It is therefore useful to continue this progress in relation to the development of Markovian-based maintenance management, which can lead to an improvement in BMSs that are already in place. A Markovian model can be used to predict the deterioration of a structure from one condition state to another over time (Roelfstra et al. 2004; Rens and Nogueria 2005; Frangopol et al. 2001). With the development of Markov chains (Markov transition matrices), predictions can be made regarding the level of future deterioration of structures. Also, with a Markov chain, the deterioration is modeled as discrete condition states rather than as a continuous deterioration process (Adey et al. 2003). Although the condition of the structure is recorded using discrete states and at discrete times using a Markovian-based methodology (unlike a reliability-based approach), the physical process of deterioration of a structure is relatively stable. Inspections and maintenance are carried out at discrete times; therefore, the state of a structure is only required at these discrete times when decisions are made (Corotis et al. 2005). The reviewed studies (Corotis et al. 2005; Cesare et al. 1992; Scherer and Glagola 1994; Jiang et al. 2000; Roelfstra et al. 2004; Orcesi and Cremona 2006) demonstrate the advances that have been made in the area of 
Markovian-based maintenance management. However, there are areas that require further research that are addressed in this paper.

It was assumed in Cesare et al. (1992) that a structural element either stays in the same condition state or deteriorates to the next lowest state in a 1-year period, and it was highlighted by Estes and Frangopol (2001) that many deterioration models that are being developed are expressed as a linear reduction in the condition state over time. A linear deterioration rate was also assumed in Mori and Ellingwood (1994). This assumption of linearity limits the ability of the methodology to consider many different materials and deterioration mechanisms. The authors have developed a method to facilitate the simulation of many forms of deterioration within a maintenance management model (i.e., gradual and abrupt growth, linear and nonlinear). This allows a wider range of materials and environments (i.e., passive and aggressive) to be studied.

Significantly, it has been recommended in the literature that Markov-chain models are further improved within a maintenance management system that takes the initiation time into consideration (Roelfstra et al. 2004). On this basis, the authors have developed a Markov-based maintenance management model that considers both the initiation phase and propagation phase of deterioration. The incorporation of the initiation phase into deterioration modeling can affect the frequency of inspections, repairs, and failures and hence the expected cost over the remaining lifetime of the structure. It is concluded that to achieve an accurate estimation of the optimal maintenance strategy and the lifetime costs of a structure, both the initiation phase and propagation phase of deterioration must be considered. The sensitivity of the results of the maintenance management model to the inclusion/length of the initiation time will be discussed as part of this study.

It was also noted that, in Macke and Higuchi (2007), when a structure was repaired, the deterioration characteristics remained the same, which implied that the repair material was the same as the construction material. This is considered to be a limitation of this methodology, because repairs of structures are most often carried out using a repair material that is different from the construction material. Therefore, as part of this work, the ability to simulate the repair of a structure using a different material (i.e., with different deterioration characteristics) will be incorporated into the methodology. However, similar to other studies (Cesare et al. 1992; Adey et al. 2003; Straub and Faber 2005; Kong and Frangopol 2003), it was decided to assume that repairs are perfect (i.e., each component returns to the as new condition following a repair) to reduce the number of possible outcomes. The ability to simulate the repair of a structure using a different material allows the optimal repair material to be determined for a particular structure or group of structures.

\section{Theoretical Development}

The initial theoretical development of a maintenance model was presented in Sheils et al. (2010). Markov transition matrices were used to simulate the growth, inspection, repair, and failure of a population of defects over time, thus providing the owner/manager of a structure with an optimal maintenance management strategy (i.e., optimal inspection interval, optimal combination of inspection techniques, etc.) based on the specific constraints/requirements of the structure being considered. The total range of defect sizes is broken into defect groups (10 groups in this case), and a record is kept each year of the number of defects within each group, similar to Scherer and Glagola (1994). Based on the growth rate and the kinetics of the growth, the probability of moving from one defect group to a larger defect group (i.e., a group with larger defects) is assessed. It is assumed that inspections are carried out every $\Delta T$ years, and when a repair is carried out, the defect is repaired to the smallest defect group.

\section{Defect Growth}

The upper-triangular part of the Markov matrix is controlled by the growth characteristics of the defects. Because the methodology was developed to be material independent, the ability to simulate many forms of deterioration was considered important. Therefore, two parameters representing (1) the growth rate, $\alpha$, and (2) the deterioration kinetics (i.e., abruptness) of the growth, $g$, were introduced to simulate the growth of a defect. The second parameter controls whether defects develop gradually and just move from one defect group to the next, or whether the growth of a defect is more abrupt, causing it to move from one group to a defect group a few sizes larger (rather than the one next to it). Therefore, this method is distinct from that described in Cesare et al. (1992), where a defect could move only from the group it was in to the group adjacent to it in one time step.

When considering a Markov process, what is of interest is to calculate the probability that a defect moves from one group to a larger group. The entries in the Markov transition matrix therefore represent the probability that a defect will stay in group $i\left(P_{i i}\right)$ or the probability that a defect will move from group $i$ to another group $j\left(P_{i j}\right.$ for $\left.j>i\right)$ in one time step. Also, in the transition matrix, $P_{i j}$ is the entry in the $i$ th row and the $j$ th column. For a finite number of groups, the sum of these probabilities must equal 1.0 (Cesare et al. 1992; Scherer and Glagola 1994). In this study, both deterioration parameters ( $\alpha$ and $g$ ) are used to calculate the entries in the Markov matrix that simulates growth only $\left(P_{i j \_ \text {GROWTH }}\right)$.

A higher growth rate means that there is a lower probability that the defect will remain in the same defect group after each time step, and a lower growth rate increases the likelihood that it will stay in the same group after the same period of time. For example, crack growth in a RC structure in an aggressive marine environment is more likely to develop at a faster rate than that of a structure inland, where the exposure to chlorides is moderate; therefore, such cracks will be more likely to move to a larger defect group within a certain time interval. To model this behavior, the diagonal of the transition matrix is controlled by $(1-\alpha)$ alone, for $0<\alpha<1$. Each row in the transition matrix must sum to 1.0. Therefore, the remaining portion of the probabilities $(\alpha)$ must be distributed between the other groups (or the cells in that row of the matrix). The ratio by which they are divided is controlled by $g$, as $g$ is used to describe the growth kinetics of the defects (Sheils et al. 2010). For example, for gradual deterioration, a value of $g=5$ can be used, whereas for a very abrupt form of deterioration, a negative value can be inputted (e.g., $g=-5)$.

To calculate the values of $P_{i j_{-} \text {GrowtH }}$ in the transition matrix using $\alpha$ and $g$, another matrix $\mathbf{G}$ is created as in Eq. (1). This is an inverse power equation that is used to calculate the values in the upper triangular portion (growth part) of the matrix. This model was chosen, because it allows values of $g=0$ and negative values of $g$ to be modeled, resulting in an increased versatility to simulate different forms of the deterioration mechanism

$$
G_{i j}=\left\{\begin{array}{cc}
\frac{1}{(j-i)^{g}} & \text { for } j>i \\
0 & \text { for } j \leq i
\end{array}\right.
$$

From this matrix, the values of the transition matrix are calculated using Eq. (2) 


$$
P_{i j_{\text {GROWTH }}}=\frac{(\alpha)\left(G_{i j}\right)}{\sum G_{i}} \text { for } j>i
$$

For a high value of $g$ (e.g., $g=5$ ), the growth of an individual defect is modeled as smooth and gradual. In this case, the defect stays in the same group or moves to the next defect group and does not readily skip groups by growing very suddenly in one time step [i.e., $P_{i, i+1} \approx \alpha$, and $P_{i j} \approx 0($ for $j>i+1)$ ]. However, using this method, it is also possible to input a value of 0 or a negative value for $g$. In the special case where $g=0, \alpha$ is divided equally between all other groups for $j>i$. Similarly, when $g$ is negative, the growth kinetics of a defect are different. For a negative value of $g$, when growth of a defect occurs, there is a higher probability that it will skip over the defect groups next to it and move to larger groups. This results in very abrupt growth.

As discussed, the use of a Markov decision process within a management system can result in certain limitations when simulating the deterioration of a structure. Therefore, this work aims to overcome some of these limitations, and the ability to simulate nonlinear deterioration propagation will be discussed in the next section. The incorporation of an initiation phase into Markovianbased deterioration modeling and the repair of structures using a different material than the original construction material will subsequently be discussed.

\section{Nonlinear Growth}

In the previous section, two deterioration parameters were introduced to simulate the growth rate and growth kinetics of a defect, allowing many forms of deterioration propagation to be considered (i.e., gradual/aggressive). In this study, it is demonstrated that both linear and nonlinear deterioration behavior can be considered by specifying the deterioration parameter $\alpha$ for each defect size/range of sizes independently. Using this method, deterioration rate parameters to be inputted into the model (e.g., $\alpha$ ) can be determined by fitting to linear or nonlinear data from laboratory or on-site testing. In Sheils et al. (2010), a growth rate was specified for the material and was assumed to be constant for each group, resulting in a linear growth rate of defects over time. This may not always be accurate, as in some cases, the growth rate can change, i.e., increasing or decreasing, as the deterioration progresses. For example, considering chloride-induced corrosion of RC, corrosion products that build up on the surface of the steel can obstruct the ingress of chloride ions and oxygen into the surface of the uncorroded steel, which can lead to a reduction in the corrosion rate. In this case, the rate of crack growth would also decrease over time. Therefore, by facilitating the input of different growth parameters for each defect group, many different forms of deterioration behavior can be simulated using the Markov matrix. A linear growth rate can still be used by inputting the same growth rate for each group. By fitting the deterioration parameters to actual curves that describe the rate of crack growth over time (which can be obtained from experimental studies or on-site tests), the deterioration of different materials can be approximated and inputted into the maintenance management model to investigate the efficiency of different construction/repair materials.

\section{Initiation Phase}

As discussed, it was also recognized that some materials can demonstrate an initiation phase and a propagation phase of deterioration. This form of deterioration is most commonly associated with corrosion of RC but is also appropriate for modeling the deterioration of other structural materials, such as coated steel. Taking RC as an example, during the initiation phase there is no corrosion of the reinforcing bars but aggressive agents (such as chlorides and carbon dioxide) ingress into the concrete cover toward the steel. It is important to model this initiation time when investigating the repair efficiency of different materials. This can affect the frequency of inspections, repairs, and failures and hence the expected cost over the remaining lifetime of the structure/network of structures. Once these aggressive agents break down the passive layer surrounding the steel, the initiation phase ends, and the propagation phase of deterioration begins. During the propagation phase, corrosion begins and causes cracking of the concrete cover. The maintenance management model has therefore been further developed to take this two-step deterioration process into account. The modeling process is illustrated in Fig. 1. However, using the developed methodology, the initiation phase can also be bypassed and set to zero for materials that do not follow this kind of deterioration behavior (i.e., materials with a propagation phase only).

The initiation time is modeled based upon the properties of the material, i.e., the original material that the structure was constructed with and the new material that the defects are repaired with. In a new structure, all defect locations (or hot spot locations) begin in the initiation phase of the original material (Fig. 1). Over time, defects grow, and inspections are carried out. When repair or failure occurs, the defects are repaired with a repair material (which can be a new material or the same as the original material).

Again, taking RC as an example, when a patch repair is carried out, all the old concrete cover is broken out and replaced with a new concrete repair material. It is assumed here that the aggressive agents such as chloride ions and carbon dioxide are completely removed, and therefore, a new initiation phase begins, while these aggressive agents again ingress into the new repair concrete. In this case, the initiation time is dictated by the properties of the repair material. Therefore, when an inspection indicates that the defect size is greater than the critical defect size $\left(d_{c}\right)$ and repairs are carried out, defects move from the defect group they were in to the beginning of the initiation phase of the new material.

To model this behavior, matrices were developed to keep a record of the number of defects in each group in each of the materials over time. The developed model assumes that in any year of simulation the inspection, repair, and failure occur at the end of the year in question (i.e., it is assumed that defects may grow throughout the year before inspections, repair or failure occur). Five new matrices were initiated to keep track of the number of defects in each group, and one matrix was initiated to keep track of the number defects in each year of the initiation phase.

- The matrix \#Defects_EndYr_BeforeFailure records the number of defects in each group in each material after the defects have grown throughout the year before the simulation of failure. This matrix has entries in the years between inspections.

- The matrix \#Defects_EndYr_AfterFailure records the number of defects in each group in each material after the defects have grown throughout the year and after failure has taken place. Therefore the failed defects are taken from the relevant groups and added to the initiation phase of the new material for the specified initiation time. This matrix has entries in the years between inspections.

- The matrix \#Defects_EndYr_BeforeRepairFailure records the number of defects in each group in each material after the defects have grown throughout the year before the simulation of repair and failure. This matrix has entries only for inspection years.

- The matrix \#Defects_EndYr_AfterRepairFailure records the number of defects in each group in each material after the defects have grown throughout the year and after repair and failure have 


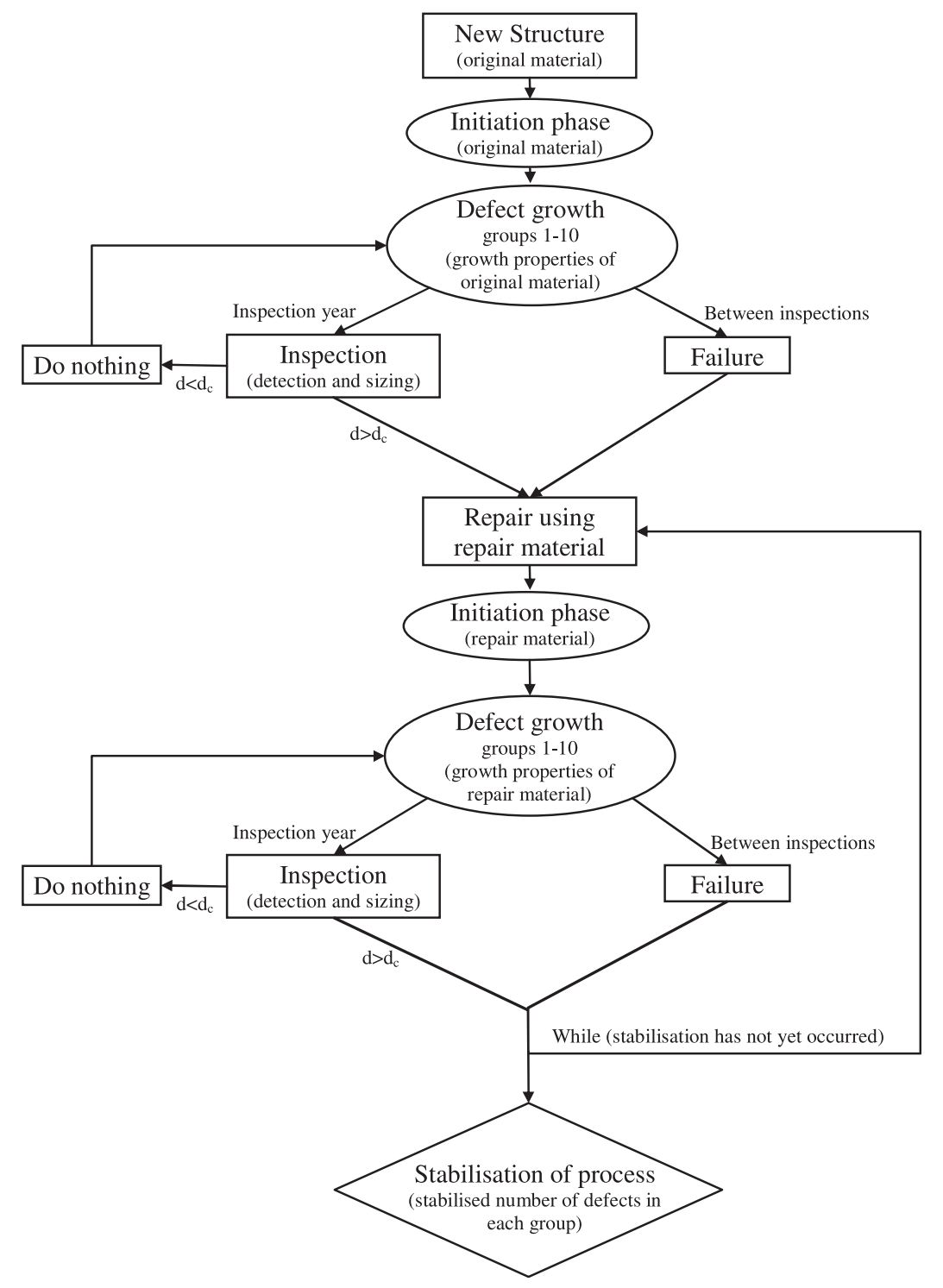

Fig. 1. Illustration of possible deterioration behavior of original material and repair material

taken place. Therefore, the repaired/failed defects are taken from the relevant groups and added to the initiation phase of the new material for the specified initiation time. This matrix has entries only for inspection years.

- The matrix \#DefectsBeginYr records the number of defects in each group in each material at the beginning of each year. This is therefore the number of defects in each group after repair and failure has taken place but also after defects have been added into the first group of the repair material once the initiation time after a repair or failure has passed. This matrix has entries each year of the simulation.

- The matrix InitiationPhase records the number of defects that are added to the initiation phase of each material over time. These defects are then added to the first group of the repair material once the initiation time has passed.

Fig. 2 illustrates the procedure for simulating the growth and failure of defects for a year of simulation $(Y)$ between inspections (i.e., $Y \neq n \Delta T$, where $n$ is a positive integer). In this case (between inspections), only four of the six matrices that were defined are used (i.e., \#Defects_EndYr_BeforeFailure, \#Defects_EndYr_ AfterFailure, \#DefectsBeginYr, and InitiationPhase). For a year of simulation where an inspection takes place (i.e., $Y=n \Delta T$ ), both repairs and failures must be considered, and the number of failures is replaced by the number of repairs/failures. In this case, the matrices \#Defects_EndYr_BeforeFailure and \#Defects_EndYr_AfterFailure are replaced with \#Defects_EndYr_BeforeRepairFailure and \#Defects_EndYr_AfterRepairFailure.

A while loop is set up to calculate the stabilized number of defects in each group (as illustrated in Fig. 1) using the relevant Markov matrix for each year (inspection year or between inspections) and for each material (original or repair material). Using the counter of the while loop, a record is kept of how many years have passed since repair or failure, and after the initiation period $\left(T_{i}\right)$, the defects are added to the first group of the repair material. Until the initiation time of the original material has passed, nothing can happen, as there are no defects that can grow, be inspected and repaired, or fail. Once the initiation phase of the original material has passed, defects in the original material gradually deplete, as they are repaired with a new material. By the time stabilization has occurred, all defects are in the new repair material. The results are therefore not sensitive to the properties of the original material. The stabilized number of defects in each 


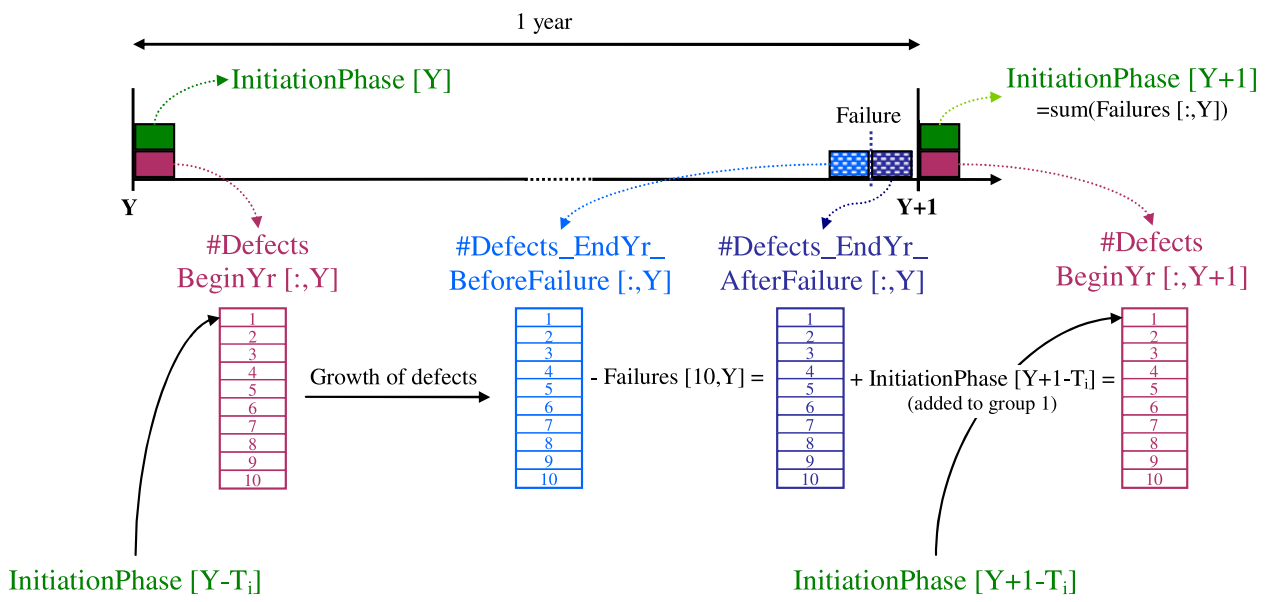

Note: Following Matlab notation, NoDefectsBeginYr [:,Y] indicates that the entries of each row of the Yth column are presented (i.e., ":" indicates all entries for the specified column of year Y)

Fig. 2. Schematic illustration of the simulation of the initiation, growth, and failure of defects between inspections

group of the repair material is used to calculate the expected mean annual number of failures for a population of defects. This allows owners/managers of structures to compare the efficiency of different repair materials (i.e., OPC, OPC + PFA, and OPC + GGBS in this case, where OPC is ordinary portland cement, $\mathrm{OPC}+\mathrm{PFA}$ is a blend of ordinary portland cement and pulverized fuel ash, and OPC + GGBS is a blend of ordinary portland cement and ground granulated blast furnace slag) in terms of both the initiation phase and the propagation phase of deterioration to determine the most effective repair strategy and subsequent inspection strategy for the structure or group of structures being considered.

\section{Failure between Inspections}

If a defect continues to grow without any repairs being carried out, failure will eventually occur. Therefore, failure must be simulated between inspections. For each defect group, the probability of failure is calculated to assess the probability that a defect will fail and subsequently be repaired, returning to the smallest defect group (i.e., $P_{i 1}$ ). It was decided here to calculate the annual probability of failure, $p_{F}$, using the Weibull cumulative distribution function based on the mean size of the defects in each group, $\bar{d}_{i}$ [Eq. (3)]. The values chosen for the reference defect size for the probability of failure $\left(d_{\text {ref-pf }}\right)$, the limit defect size $\left(d_{1}\right)$, and the Weibull shape parameter ( $m$ ) depend on the limit state and the mode of failure being considered by the owner/manager

$$
\left.p_{F}(d)=1-\left[\exp -\frac{\overline{d_{i}}-d_{1}}{d_{\text {ref_pf }}}\right)^{m}\right]
$$

\section{Repair and Failure during an Inspection Year}

Over the lifetime of a structure, inspections and repairs are carried out, and in some cases, failure can occur. However, in an inspection year, it is assumed that failure will only occur if a repair is not carried out. In this case, the probability of moving back to the initial defect group $\left(P_{i 1}\right)$ is calculated using a combination of the probability of repair and the probability of failure given that the repair has not been carried out. Using the parameters associated with the inspection techniques and the failure of the defects, the probability of repair and the probability of failure are calculated for each group. These values, along with the mean size and standard deviation of the defects in each group, are used in the calculation of the $P_{i 1}$ column for the Markov matrix simulating the behavior of a population of defects in an inspection year.

To calculate the probability of repair of defects in each group, it is necessary to assess the probability of detection (PoD), probability of false alarm (PFA), probability of good assessment (PGA), and probability of wrong assessment (PWA) for each group, as discussed in Sheils et al. (2010). There have been many studies that focus only on the detection stage of an inspection, using various sets of parameters such as PoD and PFA (Rouhan and Schoefs 2003), PoD and probability of false indications (Straub and Faber 2003), or PoD and false call probability (Chung at al. 2006) to assess the quality of a particular inspection method. In this study, a two-stage inspection process is assumed; the first inspection is carried out to detect defects present (inspection result $\widehat{d}_{1}$ ) in the structure, and the second inspection is carried out to size defects that are found to be present (inspection result $\widehat{d}_{2}$ ). In relation to detection, the PoD and PFA are estimated for each defect group, given the mean and standard deviation of the defects in the group, the detection threshold, and the quality of the inspection method being used.

It is assumed that the defect size and the noise are normally distributed and noncorrelated and that, for detection of defects, the quality (and hence cost) of the inspection method is related to the distribution of the noise (Sheils et al. 2010). If on-site calibration tests are performed for nondestructive testing (NDT) tools, distributions of noise and signal are generally available; thus, a polynomial projection of noise and signal chaos facilitates better precision for PoD and PFA (Schoefs et al. 2009). The mean value 
of the noise depends on environmental conditions and human interference and is assumed to be the same for each defect group.

Similarly, for each defect group, the values of PGA and PWA are estimated given the mean and standard deviation of the defects in the group, the critical defect size, $d_{c}$, and the quality of the inspection method being used for sizing. For sizing, there is only one distribution representing the distribution of defects and the sizing error attributable to noise. It is assumed for assessment also that the quality of the inspection method is related to the distribution of the noise. There is also a distinction made in this work between good and wrong assessments that lead to repair and those which lead to no repair (Sheils et al. 2010). This modeling allows the introduction of a risk-based definition of NDT performance in the decision scheme.

However, in an inspection year, it is assumed that failure can occur if a repair is not carried out or if it is postponed. When the first inspection (i.e., detection stage) is carried out to detect a defect, there can be two decision outcomes. One is to carry out a further assessment, and the other is to do nothing. Similarly, when a second inspection (i.e., sizing stage) is carried out to assess the size of a defect, there can also be two decision outcomes. One is to repair if the inspection indicates that the defect is larger than the critical defect size $\left(d_{c}\right)$, in which case the defect returns to the initial defect group, and the other is to carry out no repair. Failure can occur if no further assessment is carried out at the detection stage $[P($ Failure $\mid$ No assessment $)]$ or if no repair is carried out at the sizing stage $[P($ Failure $\mid$ No repair $)]$, as illustrated in Fig. 3. Similar to the event of repair, if failure occurs, the defect returns to the initial defect group. These probabilities are calculated analytically and are combined to assess $P_{i 1}$ for each defect group.

Therefore, in an inspection year, the probability of repair or failure (event combination $R \cup F$ ) for each group, $p_{R \cup F_{-} i}$, can be calculated using Eq. (4)

$$
\begin{aligned}
p_{R \cup F_{-} i}= & P_{i}(\text { Repair })+P_{i}(\text { Failure } \mid \text { No assessment }) \\
& +P_{i}(\text { Failure } \mid \text { No repair })
\end{aligned}
$$

Assuming that inspection results are independent, Eqs. (5)-(7) can be developed

$$
\begin{aligned}
P(\text { Repair }) & =\sum_{i=1}^{N} P_{i}(\text { Repair }) \\
& =\sum_{i=1}^{N} P\left(\widehat{d}_{1_{-} i} \geq d_{\min }\right) \times P\left(\widehat{d}_{2 \_i} \geq d_{c}\right)
\end{aligned}
$$

$$
\begin{aligned}
P(\text { Failure } \mid \text { No assessment }) & =\sum_{i=1}^{N} P_{i}(\text { Failure } \mid \text { No assessment }) \\
& =\sum_{i=1}^{N} P\left(\widehat{d}_{1_{-} i}<d_{\min }\right) \times p_{F_{-} i}
\end{aligned}
$$

$$
\begin{aligned}
P(\text { Failure } \mid \text { No repair })= & \sum_{i=1}^{N} P_{i}(\text { Failure } \mid \text { No repair }) \\
= & \sum_{i=1}^{N} P\left(\widehat{d}_{1_{-} i} \geq d_{\min }\right) \times P\left(\widehat{d}_{2 \_i}<d_{c}\right) \\
& \times p_{F_{-} i}
\end{aligned}
$$

where $N=$ number of defect groups (10 in Fig. 1, for example); $\widehat{d}_{1}=$ size of the defect from the detection inspection (for each group, $i$ ); $d_{\text {min }}=$ detection threshold; $\widehat{d}_{2}=$ size of the defect from the sizing assessment (for each group, $i$ ); $p_{F \_}=$probability of failure for group $i$; and $d_{c}=$ critical defect size.

In addition, the repair of a structure can be simulated using a different material than the original construction material, allowing the repair efficiency of different materials to be studied. This allows the owner/manager to consider the deterioration of the original structure and select an appropriate repair material by comparing the efficiency of different repair strategies in relation to the expected cost and optimal inspection interval. When the first defect develops in a structure, it develops according to the growth parameters assigned to the original material. As the defect grows, inspections are carried out that may lead to repair of the defect. Alternatively, failure can occur, and in this case, a repair must also be carried out.

When repair is carried out, a new more durable material may be used, and any defect that develops in the new material will develop according to the deterioration parameters of the new material. For

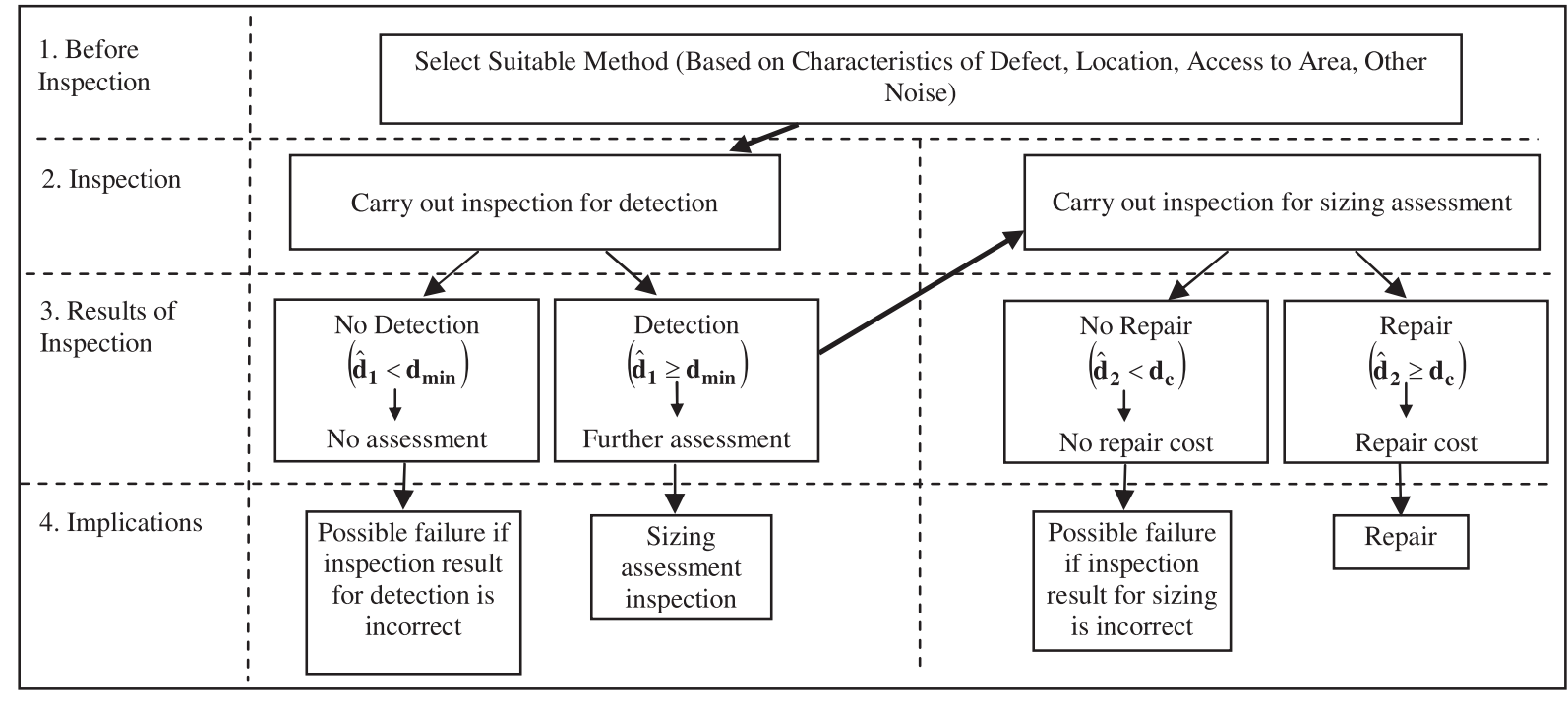

Fig. 3. Inspection outcomes for a defect group 
example, the repair material may have a longer initiation period and a slower rate of crack propagation, as illustrated in Fig. 4. Therefore, the model can be used to study the deterioration of the original material that was used to build the structure and to study the efficiency of the chosen repair material where the frequency of inspection and repair is a function of the repair material. In this study, all repairs are assumed to be carried out using the same material over the lifetime of the structure.

Therefore, in the revised model, as a defect is repaired it acquires the new properties of the repair material (i.e., initiation time, propagation rate), and a record is kept of the number of defects in each material over time. In relation to the propagation phase, a different Markov matrix is developed for each material and is used to model the growth, inspection, repair, and failure of the population of defects over time. As defects in the original material are repaired or fail, the defects are removed from the relevant group in the original material and added to the first group (or initiation phase) of the repair material. The model is run until stabilization of the number of defects in each group has occurred for each material. The total number of inspections, repairs, and failures can be calculated from the stabilized number of defects in each group, allowing the lifetime cost of a structure or group of structures to be determined. Therefore, the maintenance strategy can be optimized not only as a function of the defect deterioration mechanism of the original material but also with respect to the deterioration behavior of the chosen repair material.

\section{Stabilization of Process}

Once the Markov growth matrix for deterioration has been developed and the values for the probability of repair/failure in an

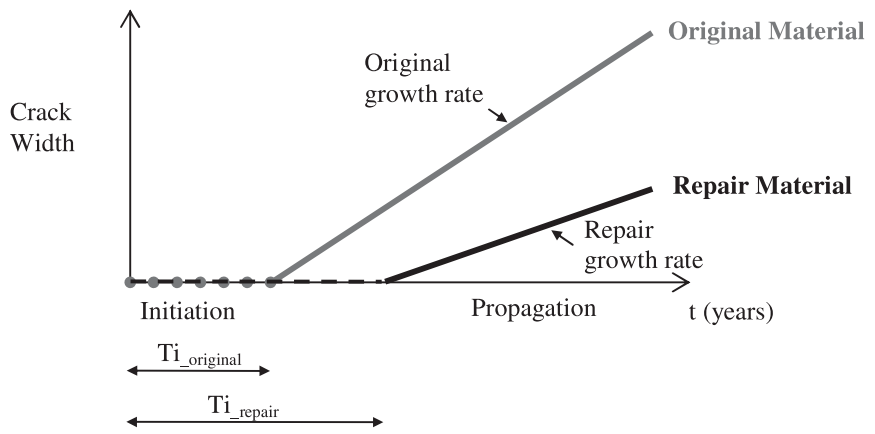

Fig. 4. Illustration of inspection, repair, and failure process of defects when repaired with a new material inspection year and the values for the probability of failure between inspections have been calculated for each group, these values need to be added to the first column of the growth matrix (whether it be for an inspection year or a year between inspections) to develop the two complete Markov matrices, which can then be used in the maintenance management model.

This Markov process is assumed to be stable; therefore, each row of the matrix must sum to 1.0, and because the entries in each of the rows in the deterioration matrix summed to 1.0 before the introduction of the $P_{i 1}$ values (which are added to simulate repair/ failure), this deterioration process must be modified to take repair and failure into account and maintain the stability of the Markov process. The methodology described assumes that in any year of simulation, the inspection, repair, and failure occur at the end of the year in question (Fig. 5). Therefore, when modifying the matrix it is assumed that defects may grow throughout the year before inspections, repair, or failure occur.

The probability that a defect in any one group will be repaired (or will fail) in one time step is represented by $P_{i 1}$. However, in one time step, before the inspection takes place the defects in each group may grow and move to larger groups, in which case the probabilities of repair or failure for the larger groups are also used to calculate the value of $P_{i 1}$ for one row in the matrix. The entries in the complete Markov matrix for an inspection year, $P_{i j \_ \text {MARKOV_1 }}$, are calculated using Eqs. (8) and (9), and the entries in the complete Markov matrix for a year between inspections, $P_{i j \_ \text {MARKOV_2 }}$, are calculated using Eqs. (10) and (11). Using this method is consistent, as the entries in each row still sum to 1.0

$$
\begin{aligned}
& P_{i 1 \_ \text {MARKOV_1 }}=P_{i 1_{\_} \text {GROWTH }}+\sum_{j=2}^{N}\left(P_{i j \_ \text {GROWTH }} \times p_{R \cup F_{-} j}\right) \\
& P_{i j_{-} \text {MARKOV }_{-} 1}=P_{i j_{-} \mathrm{GROWTH}} \times\left(1-p_{R \cup F_{-} j}\right), \quad \text { for } j>1 \\
& P_{i 1_{-} \text {MARKOV_2 }}=P_{i 1_{-} \text {GROWTH }}+\sum_{j=2}^{N}\left(P_{i j_{-} \text {GROWTH }} \times p_{F_{-j} j}\right) \\
& P_{i j \_ \text {MARKOV } \_2}=P_{i j \_ \text {GROWTH }} \times\left(1-p_{F_{-} j}\right), \quad \text { for } j>1
\end{aligned}
$$

Once both matrices have been formulated, they are used to simulate the growth, failure, and repair of a population of defects over time. Each defect group is assumed to have an initial population of defects.

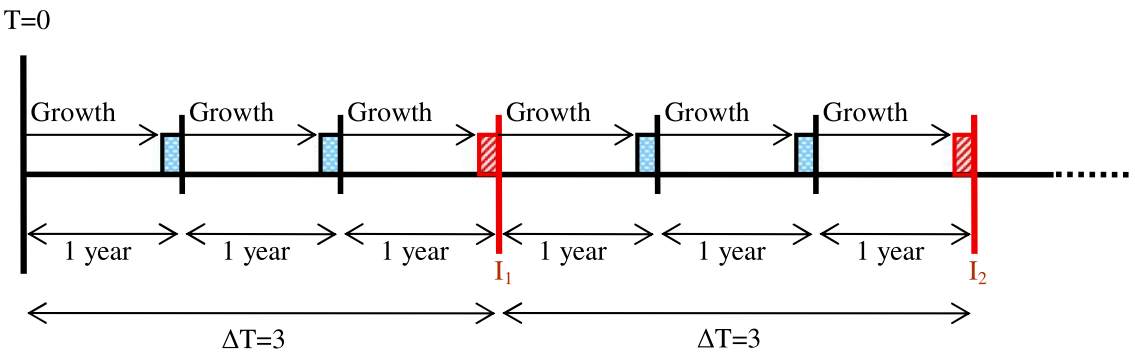

Simulation of failure
Simulation of inspection/repair/failure

Fig. 5. Illustration of the methodology for the development of the Markov matrix 
Using this methodology, the number of defects in each group is calculated on a yearly basis using the relevant Markov matrix and the number of defects in each group from the previous year. After a period of time, the number of defects in each group stabilizes and remains constant. The model is run until the number of defects in each group reaches this steady state. The stabilized number of defects in each group is then used to calculate the expected number of failures for a group of structures (Sheils et al. 2010). The number of defects in each group depends on factors such as the deterioration process, the characteristics of the concrete, the repair threshold, and the quality of the inspection methods.

\section{Practical Example}

In the following study, the effect of the inclusion of the initiation time into the Markov deterioration process is investigated. A RC structure is considered, because it is the most common construction material associated with the two-stage deterioration model of initiation and propagation. According to Tilly (2007), results indicated that corrosion is the most common form of deterioration in $\mathrm{RC}$, leading to more than $55 \%$ of the repairs carried out. Therefore, in this example, the rate of crack growth in RC attributable to chloride-induced corrosion is considered. In this study, three typical repair mixes were considered, OPC, OPC + PFA $(30 \%$ PFA), and OPC + GGBS (50\% GGBS). The ratio of the initiation time of the OPC + PFA mix and OPC + GGBS mix to the initiation time of the OPC mix was chosen as 2.0 and 2.8, respectively, based on the ratio of initiation times and diffusion coefficients presented in the literature (Schoefs et al. 2009; Dhir et al. 1994; Hussain and Rasheeduzzafar 1994). However, it is recognized that the initiation time may change depending on the properties of the material, the environment, and the concrete cover on the reinforcing bars. Therefore, to study the effect of changes in the initiation time, the rate of propagation is kept constant for each case, and the initiation time is varied. Based on information in the literature (Dhir et al. 1994; Hussain and Rasheeduzzafar 1994), the initiation time of the OPC concrete mix is assumed to vary from 0 to 15 years (in 5-year intervals). It is also assumed that the ratios of initiation time for the other repair materials in relation to the initiation time of OPC remain the same (i.e., 2.0 for OPC + PFA and 2.8 for OPC + GGBS). Table 1 presents the four cases considered as part of this sensitivity study. Based on the results of this study, owners/ managers of structures can consider the effect of incorporating and varying the initiation time on the relative performance of the different repair materials.

The rates of crack propagation for the three mixes (OPC, OPC + PFA, and OPC + GGBS) were obtained from an impressed-current accelerated-corrosion experimental study (Downey et al. 2012). Once hairline cracks developed on the slabs, two crack measurement devices were glued onto the concrete along each crack to monitor the growth of the cracks over time. The experiment was run until the crack width in each of the concrete mixes had reached at least $1.0 \mathrm{~mm}$, which is considered the limit crack width for this deterioration process (Vu 2003; Vu et al. 2005). These results were modified using a conversion factor that relates the accelerated corrosion test to reallife structures using the relative corrosion rates ( $\mathrm{Vu}$ 2003). This amended rate of crack propagation for each of the concrete mixes is presented in Fig. 6. The two modeling parameters that were introduced into the model to simulate the deterioration rate $(\alpha)$ and kinetics $(g)$ of the propagation phase were fitted to these experimental curves, as illustrated in Fig. 7. The results of this fitting exercise are presented in Table 2. In this example, 10 defect group sizes were considered ranging from 0.0 to $1.0 \mathrm{~mm}$; the sensitivity of
Table 1. Four Cases Considered in Sensitivity Study of Initiation Time

\begin{tabular}{lcccc}
\hline & \multicolumn{4}{c}{ Estimated initiation time (years) } \\
\cline { 2 - 5 } Concrete mix & Case 1 & Case 2 & Case 3 & Case 4 \\
\hline OPC & 0 & 5 & 10 & 15 \\
OPC + PFA & 0 & 10 & 20 & 30 \\
OPC + GGBS & 0 & 14 & 28 & 42 \\
\hline
\end{tabular}

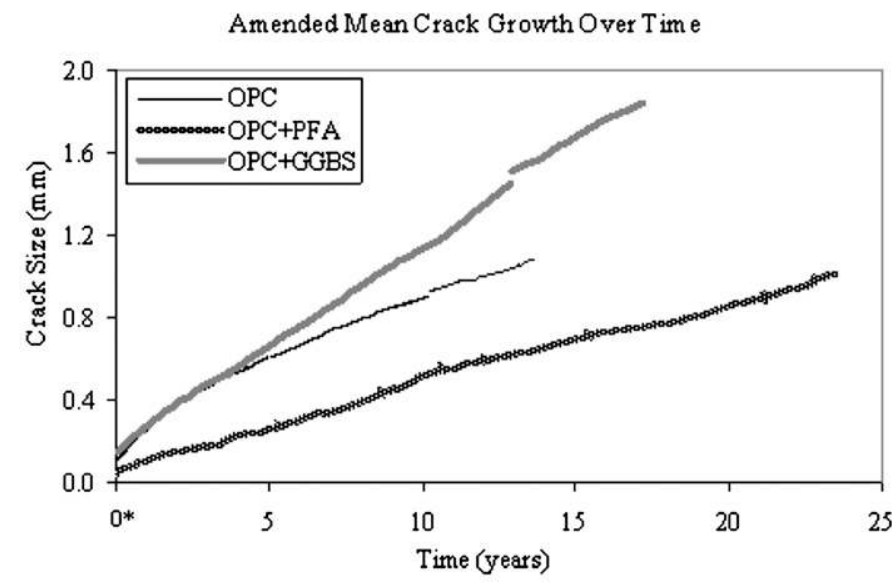

(0*time which crack me asurement devices were attached)

Fig. 6. Estimated real-time rate of crack growth for different repair materials

the model to the number of defect groups selected is studied elsewhere (Sheils et al. 2010). As indicated in Table 2, the $\alpha$-value for the 10 th (and final) group must be zero. There are no larger defect groups defined in this example, so defects cannot grow any larger once they reach Group 10.

As discussed earlier, the ability to input different growth parameters for each defect group allows nonlinear deterioration curves, like those obtained from the experimental study (for OPC), to be inputted into the maintenance management model. This allows a very good approximation of the experimental crack growth data to be achieved (i.e., with coefficient of determination values of $0.998,0.995$, and 0.999 for OPC, OPC + PFA, and OPC + GGBS, respectively), as illustrated in Fig. 7.

This methodology therefore allows owners/managers of structures to compare the relative benefits and shortcomings of different repair materials in terms of both the initiation phase and propagation phase of deterioration and to choose the most suitable repair option for a particular structure or group of structures within possible requirements/constraints.

\section{Results}

Using the varying initiation time for each of the concrete mixes and the properties of the propagation phase, the expected mean annual number of failures $[E$ (Mean annual failures)] were determined according to the methodology outlined in Sheils et al. (2010). A range of inspection intervals varying from 1 to 6 years was studied, because principal inspections are recommended every 6 years in the United Kingdom (Vassie and Arya 2006) and from 1 to 6 years in Ireland (Duffy 2004). It is recognized that the results may also be presented in terms of expected costs; however, only the expected 


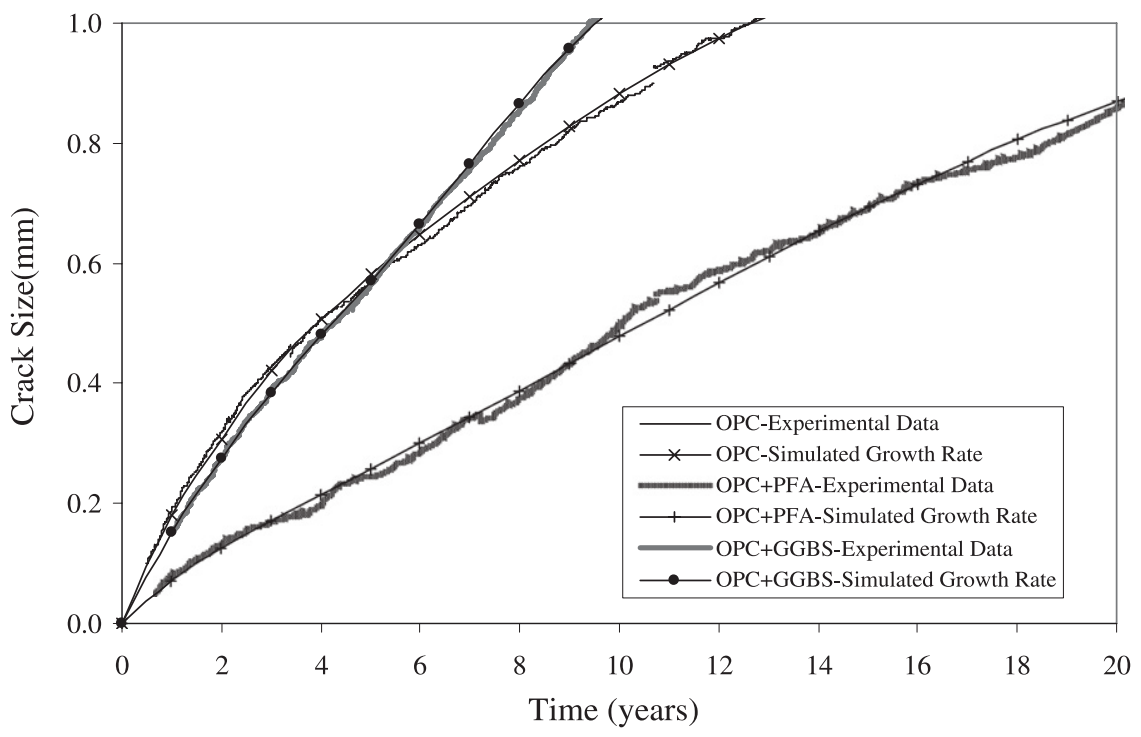

Fig. 7. Amended experimental data and simulated growth rate for crack growth

Table 2. Growth Parameters for the Different Repair Materials Fitted to Experimental Results

\begin{tabular}{lclcc}
\hline Defect group & Defect range $(\mathrm{mm})$ & OPC & OPC + PFA & OPC + GGBS \\
\hline Group 1 & $0.0-0.1$ & 1 & 0.4 & 0.85 \\
Group 2 & $0.1-0.2$ & 1 & 0.2 & 0.9 \\
Group 3 & $0.2-0.3$ & 1 & 0.3 & 0.9 \\
Group 4 & $0.3-0.4$ & 0.75 & 0.5 & 0.8 \\
Group 5 & $0.4-0.5$ & 0.6 & 0.6 & 0.6 \\
Group 6 & $0.5-0.6$ & 0.55 & 0.6 & 0.9 \\
Group 7 & $0.6-0.7$ & 0.55 & 0.4 & 1 \\
Group 8 & $0.7-0.8$ & 0.6 & 0.4 & 1 \\
Group 9 & $0.8-0.9$ & 0.6 & 0.4 & 1 \\
Group 10 & $0.9-1.0$ & 0.0 & 0.0 & 0.0 \\
\hline
\end{tabular}

Note: The value of $g$ is 3.0 for each of the different repair materials.

number of failures are considered in this case, because cost models and their associated parameters can prove subjective.

The results of this study are presented in Fig. 8 for each of the cases presented in Table 1. Based on the results of this study, for each assumed initiation time it is interesting to compare the relative merits of the different repair materials in relation to the expected number of failures. Therefore, for each case considered, the repair material that results in the lowest number of failures is highlighted in the corresponding figure.

For each material, the expected number of failures demonstrates an inverse relationship to the length of the initiation time. As the initiation time increases, the number of defects that are in the propagation phase (i.e., defect groups 1-10) declines, resulting in a lower number of defects in the larger groups where failure can occur. This in turn leads to a reduction in the expected cost. Therefore, as expected, when carrying out repairs it is worth considering increasing the initiation time by repairing defects using a material with a lower permeability by painting the concrete or by increasing the concrete cover if possible, as this will lead to a reduction in the expected number of failures over the remaining lifetime of the structure.

However, the main area of interest is the relative performance of the different repair materials as the initiation times are varied. In the first case, shown in Fig. 8(a), no initiation time is considered; therefore, the expected number of failures is influenced only by the deterioration behavior of the propagation phase for each of the materials. On this basis, OPC + PFA results in the lowest expected number of failures because of the slowest rate of crack propagation of the three concrete mixes considered. However, considering OPC and OPC + GGBS only, OPC results in the highest expected number of failures for an inspection interval of 2 years or less, whereas for larger inspection intervals, OPC + GGBS results in the highest expected number of failures (i.e., for $\Delta T>3$ years). This can be explained by studying Fig. 6 where initially OPC has a slightly higher rate of crack growth than OPC + GGBS.

In the second case, shown in Fig. 8(b), the initiation time is increased to 5 years for OPC, resulting in an initiation time of 10 and 14 years for OPC + PFA and OPC + GGBS, respectively. In this case, because of a longer initiation time (i.e., 14 years), OPC + GGBS results in the lowest expected number of failures for an inspection interval of 1 year. Because OPC + PFA has an initiation time of 10 years and the slowest rate of crack propagation, OPC + PFA results in the lowest expected number of failures for all other inspection intervals. This trend continues for Cases 3 and 4, shown in Figs. $8(\mathrm{c}$ and $\mathrm{d})$, respectively.

Because the ratios of initiation time for OPC + PFA and OPC + GGBS to OPC remain the same for each of the cases considered, as the initiation time for OPC increases, the difference between the initiation time of OPC + PFA and OPC + GGBS increases, which diminishes the superior performance of OPC + PFA, which has the slowest rate of crack propagation. For Case 3, OPC + GGBS results in the lowest number of failures for an inspection interval of 2 years or less. This improved performance of OPC + GGBS increases with the assumed initiation time for OPC, and for Case 4, OPC + GGBS has the lowest expected number of failures for an inspection interval of 3 years or less. Because there is a higher proportion of defects in the initiation phase for OPC + GGBS (because of a longer initiation period), this results in a lower number of inspections, repairs, and failures, which in turn results in a lower expected total cost.

Therefore, the importance of incorporating the initiation phase when simulating the growth repair and failure of a material with a two-stage deterioration process has been highlighted by the results of this study. The relative performance of the different repair materials also depends on the initiation phase and propagation phase of deterioration, both of which must be considered to accurately 
Case 1 (Ti_OPC $=0$ years $)$

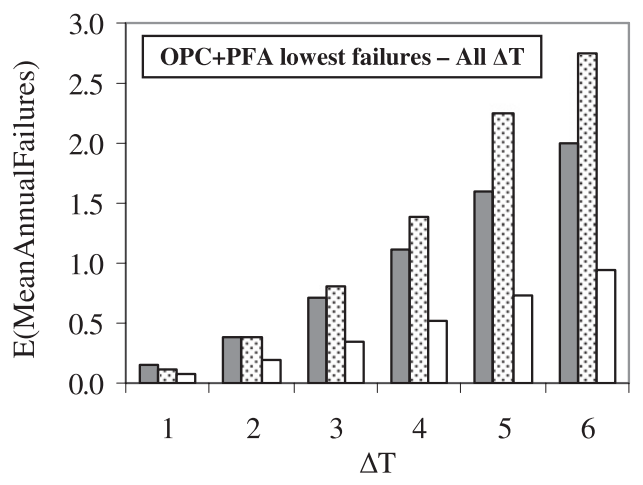

(a)

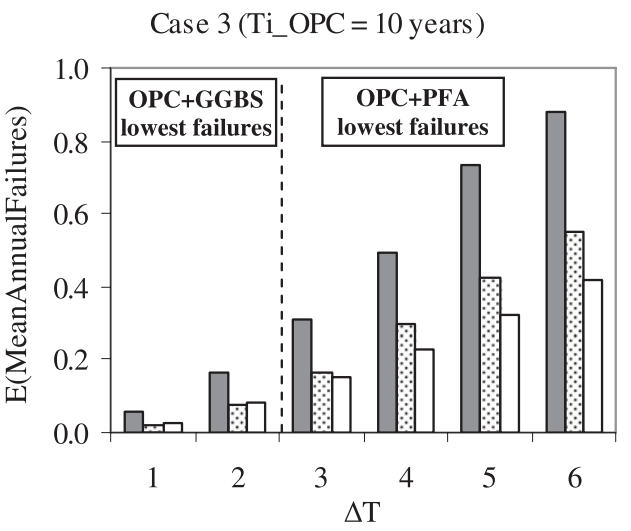

(c)

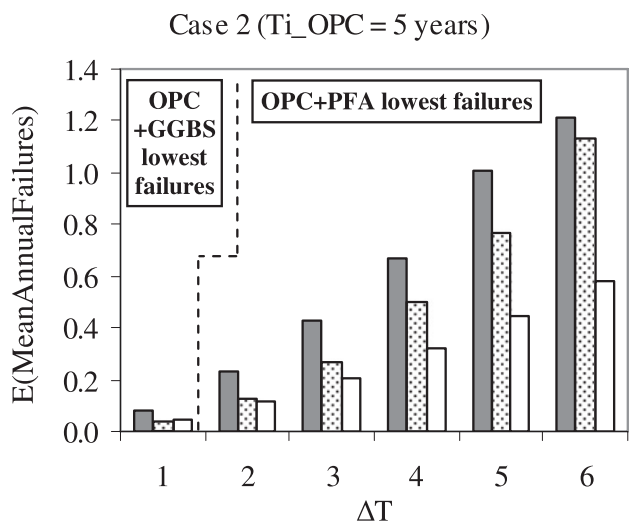

(b)

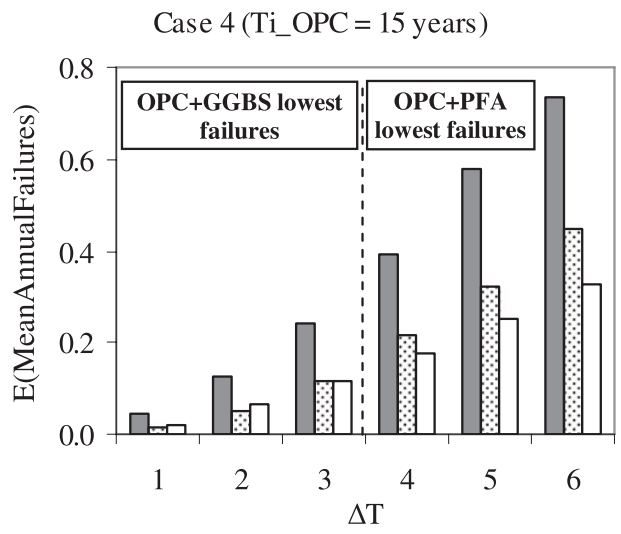

(d)

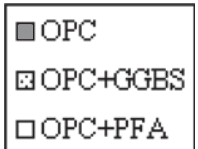

Fig. 8. Sensitivity of relative efficiency of different repair materials to initiation time

determine the optimal maintenance strategy and the lifetime costs of a structure or group of structures.

\section{Conclusions}

This paper details the advances that have been made in the further development of the Markov maintenance model that was initially presented in Sheils et al. (2010). Growth parameters can now be specified for two materials, the original material from which the structure was built and a repair material with which repairs are carried out. This allows the sensitivity of the optimal maintenance strategy to the chosen repair material to be studied. However, for this method of simulation the results are not sensitive to the properties of the original material. In addition, the ability to simulate nonlinear deterioration of defects in the propagation phase was incorporated into the methodology, allowing many different forms of defect growth and deterioration kinetics to be considered as part of the maintenance management model. More significantly, the method used to incorporate the initiation phase of deterioration into a Markov-based deterioration model was described, allowing the effect of both phases of deterioration to be investigated. In this regard, defects that are repaired are inputted into an initiation phase and re-entered into the first group of defect growth once the specified initiation period has passed. A practical example was carried out by estimating the initiation period for the different repair materials using information from the literature. For the propagation phase of deterioration, the input growth parameters needed to carry out the simulation were fitted to the experimental data to give an accurate estimation of the rate of crack growth for the different repair materials. The example illustrates how the developed methodology can be used to model the behavior of many different materials using the two-phase growth model that has been incorporated. For a material with no initiation period, the initiation time can be specified as zero.

Ultimately, the results of this study illustrate the sensitivities of the number of failures and the relative performance of the different repair materials to the assumed initiation time. As expected, an inverse relationship exists between the initiation time and the expected cost and number of failures. The variation in the expected cost with initiation time is considerable. Therefore, when repairing a structure it is worth considering designing a more durable mix with a lower permeability or increasing the concrete cover on the reinforcing steel to increase the time to initiation of corrosion. Although this may lead to a higher repair cost, it may be optimal considering the lifetime cost of a structure.

It can also be concluded that the optimal maintenance strategies and the relative performance of the different repair materials depend on both the initiation phase and propagation phase of deterioration, which highlights the importance of the incorporation of the initiation 
phase into the analysis of the optimal maintenance strategy and the lifetime cost of a structure. In addition, this section highlights how the developed methodology allows owners/managers to study the relative benefits of different repair materials considering both the initiation and propagation phases of deterioration and the sensitivity of the results to assumed parameters such as the initiation time.

\section{References}

Adey, B., Bernard, O., and Gérard, B. (2003). "Risk-based replacement strategies for deteriorating reinforced concrete pipes." Proc., 2nd Int. RILEM Workshop on Life Prediction and Aging Management of Concrete Structures, RILEM Publications, Bagneux, France 373-382.

Cesare, M. A., Santamarina, C., Turkstra, C., and Vanmarcke, E. H. (1992). "Modelling bridge deterioration with Markov chains." J. Transp. Eng., 118(6), 820-833.

Chung, H., Manuel, L., and Frank, K. H. (2006). "Optimal inspection scheduling of steel bridges using non-destructive testing techniques." J. Bridge Eng., 11(3), 305-319.

Corotis R. B., Ellis J. H., and Jiang M. (2005). "Modeling of risk-based inspection, maintenance and life-cycle cost with partially observable Markov decision processes." Struct. Infrastruct. Eng., 1(1), 75-84.

Dhir, R. K., Jones, M. R., and McCarthy, M. J. (1994). "PFA concrete: Chlorideinduced reinforcement corrosion.” Mag. Concr. Res., 46(169), 269-277.

Downey, S., Sheils, E., and O'Connor, A. (2013). "The effect of blended cements on corrosion induced cracking." Cement Concr. Res., in press.

Duffy, L. (2004). "Development of Eirspan: Ireland's bridge management system." Proc. ICE Bridge Eng., 157(3), 139-146.

Estes, A. C., and Frangopol, D. M. (2001). "Bridge lifetime system reliability under multiple limit states." J. Bridge Eng., 6(6), 523-528.

Frangopol, D. M., Kong, J. S., and Gharaibeh, E. S. (2001). "Reliabilitybased life-cycle management of highway bridges." J. Comput. Civ. Eng., 12(1), 27-34

Hussain, S. E., and Rasheeduzzafar. (1994). "Corrosion resistance performance of fly ash blended cement concrete." ACI Mater. J., 91(3), 264-272.

Jiang, M., Corotis, R. B., and Ellis, J. H. (2000). "Optimal life-cycle costing with partial observability." J. Infrastruct. Syst., 6(2), 56-66.

Kong, J. S., and Frangopol, D. M. (2003). "Life-cycle reliability-based maintenance cost optimization of deteriorating structures with emphasis on bridges.” J. Struct. Eng., 129(6), 818-828.

Liu, M., and Frangopol, D. M. (2005). "Balancing connectivity of deteriorating bridge networks and long-term maintenance cost through optimization.” J. Bridge Eng., 10(4), 468-481.
Macke, M., and Higuchi, S. (2007). "Optimizing maintenance interventions for deteriorating structures using cost-benefit criteria." J. Struct. Eng., 133(7), 925-934.

Mori, Y., and Ellingwood, B. R. (1994). "Maintaining reliability of concrete structures. II: Optimum inspection/repair.” J. Struct. Eng., 120(3), 846-862.

Neves, L. C., Frangopol, D. M., and Cruz, P. S. (2004). "Cost of life extension of deteriorating structures under reliability-based maintenance." Comput. Struc., 82(13-14), 1077-1089.

Orcesi, A., and Cremona, C. (2006). "Optimisation of reinforced concrete bridges maintenance by Markov chains." Proc., 3rd Int. Conf. on Bridge Maintenance, Safety and Management (IABMAS2006) (CD-ROM), Taylor \& Francis Group, London, 105-106.

Rens, K. L., Nogueira, C. L., and Transue, D. J. (2005). "Bridge management and non-destructive evaluation.” J. Perform. Constr. Facil., 19(1), 3-16.

Roelfstra, G., Hajdin, R., Adey, B., and Brühwiler, E. (2004). "Condition evolution in bridge management systems and corrosion-induced deterioration." J. Bridge Eng., 9(3), 268-277.

Rouhan, A., and Schoefs, F. (2003). "Probabilistic modeling of inspection results for offshore structures.” Struct. Saf., 25(4), 379-399.

Scherer, W. T., and Glagola, D. M. (1994). "'Markovian models for bridge maintenance management'.” J. Transp. Eng., 120(1), 37-51.

Schoefs, F., Clément, A., and Nouy, A. (2009). "Assessment of spatially dependent ROC curves for inspection of random fields of defects." Struct. Saf., 31(5), 409-419.

Sheils, E., O'Connor, A., Schoefs, F., Breysse, D., and Yotte, S. (2010). "Development of a two stage inspection process for assessment of deteriorating infrastructure." Reliab. Eng. Syst. Saf., 95(3), 182-194.

Straub, D., and Faber, M. H. (2003). "Modeling dependency in inspection performance." Proc., 9th Int. Conf. on Applications of Statistics and Probability in Civil Engineering (ICASP9), Millpress, Rotterdam, Netherlands, $1123-1130$.

Straub, D., and Faber, M. H. (2005). "Risk based inspection planning for structural systems." Struct. Saf., 27(4), 335-355.

Tilly, G. (2007). "The durability of repaired concrete structures." Proc., Int. Association for Bridge and Structural Engineering Symp. (IABSE) (CD-ROM), Improving Infrastructure Worldwide, International Association for Bridge and Structural Engineering, Zurich, Switzerland.

Vassie P. R., and Arya C. (2006). "Long-term maintenance strategies for highway bridges." Proc. ICE Bridge Eng., 159(2), 83-90.

$\mathrm{Vu}$, K. (2003). "Corrosion induced cracking and spatial time dependent reliability analysis of reinforced concrete structures." $\mathrm{Ph} . \mathrm{D}$. thesis, Univ. of Newcastle, Newcastle, Australia.

Vu, K., Stewart, M. G., and Mullard, J. A. (2005). "Corrosion-induced cracking: Experimental data and predictive models." ACI Struct. J., 102(5), 719-726. 